\title{
Front Matter: Volume 9753
}

, "Front Matter: Volume 9753," Proc. SPIE 9753, Optical Interconnects XVI, 975301 (25 May 2016); doi: 10.1117/12.2240147

SPIE. Event: SPIE OPTO, 2016, San Francisco, California, United States 


\title{
PROCEEDINGS OF SPIE
}

\section{Optical Interconnects XVI}

\author{
Henning Schröder \\ Ray T. Chen \\ Editors
}

15-17 February 2016

San Francisco, California, United States

Sponsored and Published by

SPIE 
The papers in this volume were part of the technical conference cited on the cover and title page. Papers were selected and subject to review by the editors and conference program committee. Some conference presentations may not be available for publication. Additional papers and presentation recordings may be available online in the SPIE Digital Library at SPIEDigitallibrary.org.

The papers reflect the work and thoughts of the authors and are published herein as submitted. The publisher is not responsible for the validity of the information or for any outcomes resulting from reliance thereon.

Please use the following format to cite material from these proceedings:

Author(s), "Title of Paper," in Optical Interconnects XVI, edited by Henning Schröder, Ray T. Chen, Proceedings of SPIE Vol. 9753 (SPIE, Bellingham, WA, 2016) Six-digit Article CID Number.

ISSN: 0277-786X

ISSN: 1996-756X (electronic)

ISBN: 9781628419887

Published by

SPIE

P.O. Box 10, Bellingham, Washington 98227-0010 USA

Telephone +1 3606763290 (Pacific Time) · Fax +1 3606471445

SPIE.org

Copyright (C) 2016, Society of Photo-Optical Instrumentation Engineers.

Copying of material in this book for internal or personal use, or for the internal or personal use of specific clients, beyond the fair use provisions granted by the U.S. Copyright Law is authorized by SPIE subject to payment of copying fees. The Transactional Reporting Service base fee for this volume is $\$ 18.00$ per article (or portion thereof), which should be paid directly to the Copyright Clearance Center (CCC), 222 Rosewood Drive, Danvers, MA 01923. Payment may also be made electronically through CCC Online at copyright.com. Other copying for republication, resale, advertising or promotion, or any form of systematic or multiple reproduction of any material in this book is prohibited except with permission in writing from the publisher. The CCC fee code is 0277-786X/16/\$18.00.

Printed in the United States of America.

Publication of record for individual papers is online in the SPIE Digital Library.

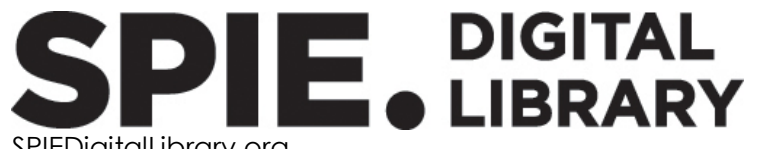

SPIEDigitalLibrary.org

Paper Numbering: Proceedings of SPIE follow an e-First publication model. A unique citation identifier (CID) number is assigned to each article at the time of publication. Utilization of CIDs allows articles to be fully citable as soon as they are published online, and connects the same identifier to all online and print versions of the publication. SPIE uses a six-digit CID article numbering system structured as follows:

- The first four digits correspond to the SPIE volume number.

- The last two digits indicate publication order within the volume using a Base 36 numbering system employing both numerals and letters. These two-number sets start with 00, 01, 02, 03, $04,05,06,07,08,09,0 A, 0 B$... 0Z, followed by 10-1Z, 20-2Z, etc. The CID Number appears on each page of the manuscript. 


\section{Contents}

vii Authors

ix Conference Committee

SESSION 1 NOVEL OPTICAL WAVEGUIDE AND INTERCONNECT TECHNOLOGIES

975302 GI-core polymer waveguide based on polynorbornene for optical interconnection (Invited Paper) [9753-1]

$975304 \quad$ High-bandwidth and low-loss multimode polymer waveguides and waveguide components for high-speed board-level optical interconnects (Invited Paper) [9753-3]

975306 Comparison of self-written waveguide techniques and bulk index matching for low-loss polymer waveguide interconnects [9753-5]

\section{SESSION 2 NANOPHOTONICS FOR OPTICAL INTERCONNECTS}

975307 A chip scale optical Tx/Rx based on silicon photonics from views of multi-mode transmission (Invited Paper) [9753-6]

975308 Silicon photonics for $100 \mathrm{Gbit} / \mathrm{s}$ intra-data center optical interconnects (Invited Paper) [9753-7]

975309 Silicon germanium on graded buffer as a new platform for optical interconnects on silicon [9753-8]

9753 OA Low latency, area, and energy efficient Hybrid Photonic Plasmonic on-chip Interconnects (HyPPI) [9753-9]

9753 OB An efficient total-internal-reflection optical switch based on reverse breakdown of pn junction and thermo-optic effect in silicon [9753-10]

\section{SESSION 3 HYBRID INTEGRATED OPTICAL LINK MODULES}

9753 OE Extended temperature performance of 120 Gbps midboard optical engine [9753-13]

9753 OG Scalable electro-photonic integration concept based on polymer waveguides [9753-50]

SESSION 4 ELECTRICAL-OPTICAL PCB TECHNOLOGIES

9753 Ol Planar polymer and glass graded index waveguides for data center applications [9753-16] 
9753 0J Electro-optical circuit board with single-mode glass waveguide optical interconnects [9753-17]

9753 OK Assembly and performance of silicone polymer waveguides (Invited Paper) [9753-18]

\section{SESSION 5 OPTICAL INTERCONNECT DEVICES AND MODULATORS}

9753 OM Light emitters and modulators on SOI for optical interconnects (Invited Paper) [9753-20]

9753 OP High quality factor trapezoidal subwavelength grating waveguide micro-ring resonator [9753-23]

$97530 Q \quad$ Miniature mid-infrared thermooptic switch with photonic crystal waveguide based siliconon-sapphire Mach-Zehnder interferometers [9753-24]

\section{SESSION 6 FIBER OPTICS AND MICRO-OPTICS INTEGRATION}

9753 OR Multimode and single-mode fibers for data center and high-performance computing applications (Invited Paper) [9753-25]

9753 OS Integration of $150 \mathrm{Gbps} /$ fiber optical engines based on multicore fibers and 6-channel VCSELS and PDs [9753-26]

9753 OT Analysis of multi-mode to single-mode conversion at $635 \mathrm{~nm}$ and $1550 \mathrm{~nm}$ [9753-28]

\section{SESSION 7 OPTICAL INTERCONNECT SYSTEMS}

9753 OV Exabits/s integrated photonic interconnection technology for flexible data-centric optical networks (Invited Paper) [9753-29]

9753 OW High-performance flat data center network architecture based on scalable and flowcontrolled optical switching system (Invited Paper) [9753-30]

9753 OY Printed polymer photonic devices for optical interconnect systems [9753-33]

\section{SESSION 8 PICS FOR OPTICAL INTERCONNECTS}

975310 SiN-assisted flip-chip adiabatic coupler between SiPh and Glass OPCBs [9753-35]

975311 Bimorph actuators in thick $\mathrm{SiO}_{2}$ for photonic alignment [9753-36]

975312 Experimental study of silicon ring resonators and ultra-low losses waveguides for efficient silicon optical interposers [9753-38]

975313 Reduced nonlinearities in 100-nm high SOI waveguides [9753-37] 
975315 Low-power chip-level optical interconnects based on bulk-silicon single-chip photonic transceivers [9753-40]

975316 Characterizations of InAs quantum dot lasers butt-joint coupled with silicon photonics waveguides [9753-41]

975317 Wavelength-controlled external-cavity laser with a silicon photonic crystal resonant reflector [9753-42]

975318 Low-loss curved subwavelength grating waveguide based on index engineering [9753-43]

POSTER SESSION

975319 Suspended mid-infrared fiber-to-chip grating couplers for SiGe waveguides [9753-44]

9753 1A A new design for coupling light between silicon strip waveguide and plasmonic slot waveguide [9753-45]

9753 1B SWG-designed MMI waveguides for dual and multi-beam splitting, beam position-shifting, and focusing purposes [9753-46]

9753 1C High performance Ge-on-Si avalanche photodetector [9753-47]

9753 1D Composite axilens-axicon diffractive optical elements for generation of ring patterns with high focal depth [9753-48]

9753 IF Full-mesh optical backplane with standard MM fiber ribbons [9753-27] 
Proc. of SPIE Vol. $9753975301-6$

Downloaded From: https://www.spiedigitallibrary.org/conference-proceedings-of-spie on 26 Apr 2023 Terms of Use: https://www.spiedigitallibrary.org/terms-of-use 


\section{Authors}

Numbers in the index correspond to the last two digits of the six-digit citation identifier (CID) article numbering system used in Proceedings of SPIE. The first four digits reflect the volume number. Base 36 numbering is employed for the last two digits and indicates the order of articles within the volume. Numbers start with $00,01,02,03,04,05,06,07,08,09,0 A, 0 B . .0 Z$, followed by 10-1Z, 20-2Z, etc.

Abdolahi, Z., 1B

Al-Saadi, Aws, 08

Apostolopoulos, Dimitrios, 10

Arndt-Staufenbiel, Norbert, OT

Atif, Muhammad, 08

Avramopoulos, Hercules, 10

Badaway, Abdel-Hameed A., OA

Ballabio, Andrea, 09

Bamiedakis, N., 04

Bansal, Lalit, OS

Basile, V., IF

Baskiotis, Catherine, 10

Baumer, Aaron M., OE

Bernabe, S., 12, 13

Bhattacharya, Shanti, 1D

Bickham, Scott R., OR

Binh, Le Nguyen, OV

Blampey, B., 12

Boersma, A., OG

Boffi, P., IF

Bogatzki, Angelina, OT

Bosman, E., OG

Boutami, Salim, 19

Bronzi, Danilo, 08

Brunner, R., 1D

Brusberg, Lars, Ol, 0J, 10

Burrell, Derek, 06

Çağrı Ulusoy, Ahmet, OS

Calabretta, Nicola, OW

Chaisakul, Papichaya, 09

Chakravarty, Swapnajit, $O Q$

Chen, J., 04

Chen, Ray T., OP, OQ, OY, 18

Chung, Chi-Jui, OQ

Corbett, B., OG

Cornelius, Joshua R., OE

Coudevylle, Jean Rene, 09

Coviello, D., IF

Cristiani, I., 13

Daly, A., OG

Dangel, R., OG

Debnath, Kapil, 17

Dharmavarapu, Raghu, ID

Dorren, Harm, OW

Dorrestein, S., OG

Duan, Guang-Hua, 19

Dubray, O., 12

Duis, J., OG

Durantin, Cédric, 19

Edmunds, James, OS

Eichhammer, Yann, 0J
Eichhorn, Catherine M., OE

El-Ghazawi, Tarek, OA

Falcucci, M., $1 \mathrm{~F}$

Fan, D. L., OP, 18

Fassi, I., $1 \mathrm{~F}$

Favreau, Julien, 19

Fédéli, Jean-Marc, 19

Ferrario, M., $1 \mathrm{~F}$

Fournier, M., 12, 13

Fowler, D., 12

Frey, Christopher, 0J

Frigerio, Jacopo, 09

Gindre, P., 12

Giuliani, G., 13

Gonzalez-Fernandez, A. A., 17

Grehn, Moritz, 08

Grellier, E., 12

Grüner-Nielsen, Lars, OS

Guo, L. Jay, OY

Guo, Wei, 16

Gustavsson, Johan, OS

Hagihara, Yasuhiko, 07

Håkansson, Andreas, 0J

Harmsma, P., OG

Hazell, John F., OE

Heikkinen, Veli, OS

Henniges, Marvin, 08

Herbst, Christian, 0J

Hodge, Malcolm H., OK

Hofmann, Jens, OT

Horimoto, Akihiro, 02

Isella, Giovanni, 09

Ishigure, Takaaki, 0

Jang, Ki-Seok, 15, 1C

Jiang, $\mathrm{H}_{\text {., }} \mathrm{BB}$

Jin, Muhyun, $O B$

Joo, Jiho, 15, 1C

Justice, J., OG

Kalavrouziotis, Dimitrios, 10

Kaminska, B., 1B

Karppinen, Mikko, OG, OS

Kaynak, Mehmet, 08

Kehayas, Efstratios, OS

Kim, Gyungock, 15, 1C

Kim, In Gyoo, 15, 1C

Kim, Jong-Hun, OB

Kim, Sanggi, 15

Kim, Sanghoon, 15, 1C

Kim, Seong-Hwan, OB

Kim, Sun $\mathrm{Ae}, 15,1 \mathrm{C}$

King, Roger, OS 
Kinoshita, Ryota, 02

Kissinger, Dietmar, OS

Kitazoe, Katsuma, 02

Ko, Minsu, OS

Korhonen, T., OG

Kozlovsky, William J., OE

Kupijai, Sebastian, 08

Kurata, Kazuhiko, 07

Kwack, Myung-Joon, 15

Lacava, C., 13

Langsam, David P., OE

Larsson, Anders, OS

Lee, Chi-Sen, 16

Lee, Dong-Wook, OB

Leinse, Arne, 11

Lemonnier, O., 12

Le Roux, Xavier, 09

Li, Qiaochu, OY

Liles, Alexandros A., 17

Lischke, Stefan, 08

Liu, Ruili, OM

Lostutter, Calob K., OK

Lutz, Sharon M., OE

Mai, Andreas, 08

Manessis, Dionysios, 0J

Marchetti, R., 13

Marrapode, Thomas R., OK

Marris-Morini, Delphine, 09

Martinelli, M., IF

Meister, Stefan, 08

Miao, Wang, OW

Middlebrook, Christopher, 06

Minzioni, P., 13

Mitcheltree, Thomas J., OE

Myöhänen, Petri, OS

Nakamura, Takahiro, 07

Narayana, Vikram, OA

Neitz, Marcel, Ol, OJ

Ning, Gordon Liu, OV

O'Faolain, Liam, 17

Offrein, B. J., OG

Ogura, Ichiro, 07

Oh, Jin Hyuk, 15, $1 \mathrm{C}$

Ollila, Jyrki, OS

Oppermann, Hermann, OJ

Ortsiefer, M., OG

Otte, Sven, 08

Pan, Zeyu, OY

Park, Hyo-Hoon, OB

Park, Hyundai, 15

Park, Jaegyu, 15

Penty, R. V., 04

Pernthaler, Dominik, 0J

Persheyev, Saydulla, 17

Peters, Tjitte-Jelte, 11

Pitwon, Richard, ol

Postma, Ferry, 11

Poulopoulos, Giannis, 10

Prak, Albert, 11

Preble, Stefan, 16

Qiu, Ciyuan, $0 M$

viii

Queisser, Marco, 0J
Reboud, V., 12

Renghini, C., $1 \mathrm{~F}$

Rhee, Hanjo, 08

Richter, Harald H., 08

Safaisini, Rashid, OS

Salminen, Noora, OS

Scalmati, Paolo, IF

Schild, Beatrice, OJ

Schröder, Henning, OI, OJ, OT, 10

Schwartz, Erik, 08

Selicke, David, 08

Sirbu, Bogdan, OJ

Sorger, Volker J., OA

Stampoulidis, Leontios, OS

Stolarek, David, 08

Su, Yikai, OM

Subbaraman, Harish, OY

Sun, Shuai, OA

Suzuki, Yasuyuki, 07

Swatowski, Brandon W., OK

Tanskanen, Antti, OS

Tao, Thomas Wang, OV

Tapaninen, Olli, OS

Taunay, Thierry, OS

Tekin, Tolga, OJ

Theiss, Christoph, 08

Thonnart, Y., 12

Tichem, Marcel, 11

Tsang, H. K., IA

Vakarin, Vladyslav, 09

Van Steenberge, G., OG

Verdiell, Jean-Marc A., OE

Vijayakumar, A., ID

Vitali, Marco, 08

Vitali, V., 13

Vivien, Laurent, 09

Wang, Kai, Ol

Wang, Yaoguo, OP, 18

Wang, Zheng, OP, 18

Wang, Zihao, 16

Weber, Daniel, 0J

Weidner, W. Ken, OK

Westbergh, Petter, OS

White, I. H., 04

Wiegersma, S., OG

Wöhrmann, Markus, $0 \mathrm{~J}$

Wörhoff, Kerstin, 11

Worrall, Alex, ol

Wu, Kai, 11

XU, Xiaochuan, OP, 18

Yamauchi, Akira, 0

Yao, Ruizhe, 16

Yashiki, Kenichiro, 07

Yeo, Sanggu, OB

Yoo, Dong-Eun, OB

Zamora, Vanessa, OT

Zbinden, Eric J., OE

Zhang, Cheng, OY

Zhang, Yong, OM

Zhu, Bingqing, $1 \mathrm{~A}$

Zimmermann, Lars, 08

Zou, Yi, OQ 


\title{
Conference Committee
}

\author{
Symposium Chairs
}

Jean-Emmanuel Broquin, IMEP-LAHC (France)

Shibin Jiang, AdValue Photonics, Inc. (United States)

Symposium Co-chairs

David L. Andrews, University of East Anglia (United Kingdom)

Alexei L. Glebov, OptiGrate Corporation (United States)

2016 Program Track Chair

Yakov Sidorin, Quarles \& Brady LLP (United States)

Conference Chairs

Henning Schröder, Fraunhofer-Institut für Zuverlässigkeit und Mikrointegration (Germany)

Ray T. Chen, The University of Texas at Austin (United States)

\section{Conference Program Committee}

Bill Blubaugh, US Conec Ltd. (United States)

Patrick B. Chu, Sandia National Laboratories (United States)

Alan F. Evans, Corning Incorporated (United States)

Alexei L. Glebov, OptiGrate Corporation (United States)

Ruth Houbertz, Multiphoton Optics GmbH (Germany)

Marika P. Immonen, TTM Technologies, Inc. (Finland)

Takaaki Ishigure, Keio University (Japan)

Wei Jiang, Rutgers, The State University of New Jersey (United States)

Mikko Karppinen, VTT Technical Research Center of Finland (Finland)

Christian Koos, Karlsruher Institut für Technologie (Germany)

Bert-Jan Offrein, IBM Research - Zürich (Switzerland)

Hyo-Hoon Park, KAIST (Korea, Republic of)

Nikos Pleros, Aristotle University of Thessaloniki (Greece)

Richard C. Pitwon, Xyratex Technology Ltd. (United Kingdom)

Richard Soref, University of Massachusetts Boston (United States)

David J. Thomson, University of Southampton (United Kingdom)

Peter Van Daele, Ghent University (Belgium)

Alan X. Wang, Oregon State University (United States)

Michael R. Wang, University of Miami (United States)

Ian H. White, University of Cambridge (United Kingdom)

Chris Q. Wu, Corning Incorporated (United States)

Xiaochuan Xu, Omega Optics, Inc. (United States) 


\section{Session Chairs}

1 Novel Optical Waveguide and Interconnect Technologies

Henning Schröder, Fraunhofer-Institut für Zuverlässigkeit und Mikrointegration (Germany)

2 Nanophotonics for Optical Interconnects

Ray T. Chen, The University of Texas at Austin (United States)

3 Hybrid Integrated Optical Link Modules

Alan F. Evans, Corning Incorporated (United States)

4 Electrical-Optical PCB Technologies

Marika P. Immonen, TTM Technologies, Inc. (Finland)

5 Optical Interconnect Devices and Modulators

Nikos Pleros, Aristotle University of Thessaloniki (Greece)

6 Fiber Optics and Micro-Optics Integration

Bill Blubaugh, US Conec Ltd. (United States)

7 Optical Interconnect Systems

Richard C. Pitwon, Seagate Systems (UK) Ltd. (United Kingdom)

$8 \quad$ PICs for Optical Interconnects

Alan X. Wang, Oregon State University (United States)

9 Hybrid Device Integration Approaches for Silicon Photonics Chips

Patrick B. Chu, Sandia National Laboratories (United States) 